Artigo Original

\title{
Comparação de modelos de regressão entre variáveis quantitativas e categóricas nos estudos de qualidade de vida de idosos
}

\author{
Comparison of regression models between quantitative and categorical variables in the studies on \\ the quality of life of the elderly \\ Mariano Martinez Espinosa' \\ Nathany Luzia de Oliveira' \\ Dayane de Carvalho Rodrigues" \\ Bryan Mariano Martínez Alves ${ }^{\prime \prime \prime}$ \\ Samira Reschetti Marcon'
}

\section{Resumo}

O objetivo do presente estudo foi avaliar o efeito das variáveis socioeconômicas, aspectos de saúde e dos domínios do WHOQOL-BREF e WHOQOL-OLD na qualidade de vida global dos idosos por meio de dois modelos estatísticos: regressão linear múltiplo quantitativo e regressão de Poisson múltiplo categórico. As análises de regressão realizadas neste estudo foram baseadas em dados de um estudo transversal, com a população de idosos usuários de quatro Centros de Convivência para Idoso no Município de Cuiabá-MT, considerando uma amostra aleatória de 317 idosos. Geralmente em estudos de qualidade de vida é aplicado o modelo de regressão linear múltiplo quantitativo, no entanto os escores desta variável frequentemente não seguem um comportamento linear, mesmo realizando uma transformação dos dados da variável resposta. Nesta situação e também considerando que este estudo é transversal, uma alternativa foi aplicação do modelo de regressão de Poisson múltiplo com os escores da variável dependente categorizados. Neste estudo, apenas o modelo Poisson múltiplo foi adequado aos dados e com ele foi constatado que as variáveis que conjuntamente apresentaram maiores riscos na qualidade de vida foram: percepção de saúde doente, não realiza caminhada e escores dos domínios físico e psicológico; todos menores que a mediana.

Palavras-chave: Qualidade de vida; Idoso; Regressão linear; Poisson

\section{Abstract}

The aim this study was to evaluate the effect of socioeconomic and health aspects variables and WHOQOL-BREF and WHOQOL-OLD domains on the overall quality of life of the elderly by two statistical models: multiple linear regression quantitative and multiple categorical Poisson regression. The regression analyzes performed in this study were based on data from a cross-sectional study with the population of elderly users from four Living Center for Senior Citizens in the Municipality of Cuiabá-MT, considering a random sample of 317 elderly. Generally, the quantitative linear regression model is applied in quality of life studies, however, the scores of this variable do not often follow a linear behavior, even when performing a transformation of the data of the response variable. In this situation and also considering that this study is transversal, an alternative was the application of the multiple Poisson regression model with the categorized dependent variable scores. In this study, only the multiple Poisson model was adequate to the data and it was found that the variables that together presented the greatest risks in quality of life were: perception of ill health, no walking and scores of the physical and psychological domains; all below than the median.

Keywords: Quality of life; Elderly; Linear; Poisson regression

Universidade Federal de Mato Grosso, Brasil Email: marianomphd@gmail.com; nathany.oliveiraO6@gmail.com; samira.marcon@gmail.com

' Centro Universitário de Várzea Grande-MT, Brasil Email: dayane.fisiot@gmail.com

I" Universidade de São Paulo, Brasil. Email: mmsbryan@hotmail.com 


\section{Introdução}

O envelhecimento é um processo natural, ativo, progressivo e irreversível da vida, acompanhado de mudanças físicas, fisiológicas e psicológicas, que ocorre ao longo do tempo em todo organismo vivo (TAVARES et al., 2016). Particularmente o envelhecimento humano é um processo complexo e multifatorial, influenciado por diferentes fatores, tais como gênero, padrões de saúde individual e coletiva da sociedade, classe social, cultura, entre outros (SCHNEIDER; IRIGARY, 2008).

O processo do envelhecimento está causando mudanças profundas em todos os setores da sociedade, mas é na saúde que têm maior preocupação, tanto por seu impacto em todos os níveis de atenção como a necessidade de novos recursos e estruturas (OLIVEIRA et al., 2014).

Assim, diante desta realidade inquestionável, evidencia-se a importância de garantir aos idosos um envelhecimento saudável e ativo com a manutenção de boa qualidade de vida (TRENTINI, 2004). Para essa finalidade no Brasil existem serviços de atenção ao idoso de grande importância, destacando-se o Centro de Convivência para Idosos (CCI), de acordo com o disposto no Decreto n ${ }^{\circ}$ 1.948, de 3 de julho de 1996. O CCI é uma modalidade não asilar de atendimento destinado à permanência diurna do idoso, onde são desenvolvidas atividades físicas, laborativas, recreativas, culturais, associativas e de educação para a cidadania (BRASIL, 1996). Segundo JORGE (2008), o CCI visa resgatar as capacidades adaptativas do idoso para que ele possa usufruir de forma permanente do intercâmbio de relações com a sua família e a sociedade. Deve se constituir num espaço de interação e encontros, e propiciar ao idoso uma reintegração sócio-político-cultural, a partir de ações integradas de diversos profissionais qualificados, numa proposta interdisciplinar para a promoção do envelhecimento. O resultado do trabalho coletivo desenvolvido no CCI é de grande importância e constitui relevante alternativa para a ressocialização, melhoria da qualidade de vida e redefinição de seu lugar na sociedade (DAL RIO, 2009).

A Organização Mundial da Saúde (OMS) define qualidade de vida como "a percepção do indivíduo de sua posição na vida, no contexto da cultura e sistemas de valores nos quais vive e em relação aos seus objetivos, expectativas, padrões e preocupações" (WHOQOL GROUP, 1995). É um conceito abrangente e complexo, que engloba a saúde física, o estado psicológico, o nível de independência, as relações sociais, as crenças pessoais e a relação com o meio ambiente (WHO, 1998; RODRIGUES, 2013).

A qualidade de vida na velhice é de grande relevância na realidade brasileira, pois existe uma nova sensibilidade tanto na visão da saúde como na atuação social, que é considerada como um problema ou como um desafio para os indivíduos e para a sociedade (GUTIERREZ et al., 2011). No entanto, a qualidade de vida do idoso pode ser afetada por diversos fatores tais como: o sexo, idade, aspectos de saúde, condições econômicas, psicológicas, funcionais, sociais entre outros (SANTOS et al., 2007). Para avaliar os efeitos que esses fatores exercem sobre a variável qualidade de vida e quantificar a grandeza desses efeitos, geralmente são utilizadas técnicas estatísticas de análise de regressão por meio de diversos modelos. Considerando como variável dependente a qualidade de vida global (QVG) e/ou os domínios de qualidade de vida e/ou as facetas especificas de cada domínio (SPILKER, 1990).

Em muitos trabalhos de qualidade de vida geralmente é aplicado o modelo de regressão linear múltiplo quantitativo (PEREIRA et al. 2006; TAVARES et al. 2012; VAGETTI et al. 2012), uma vez que os escores da variável dependente usualmente é expressa em porcentagem. No entanto, os valores desses escores muitas vezes não seguem um comportamento linear, mesmo realizando uma transformação nos dados da variável dependente. Nesta situação, a utilização deste modelo pode levar a conclusões equivocadas das inferências realizadas.

$\mathrm{Na}$ ausência de linearidade na variável dependente e também em estudos transversais, uma alternativa é a aplicação do modelo de regressão de Poisson múltiplo com os escores da variável dependente categorizados. Neste caso, para categorizar os escores, pode ser utilizada a mediana como ponto de corte.

Portanto, o objetivo do presente estudo foi avaliar o efeito das variáveis socioeconômicas, aspectos de saúde e dos domínios do WHOQOL-BREF e WHOQOL-OLD (FLECK, 1998) na qualidade de vida global dos idosos por meio de dois modelos estatísticos: regressão linear múltiplo quantitativo e regressão de Poisson múltiplo categórico.

\section{Material e método}

\section{Descrição dos dados}

\subsubsection{Tipo, população e local de estudo}

As análises de regressão realizadas no presente estudo foram baseadas em dados de um estudo transversal, realizado pela pesquisadora Rodrigues (2013) do Programa da Pós-Graduação do Instituto de Saúde Coletiva da Universidade Federal de Mato Grosso, no período de julho a dezembro de 2012 , com a população de idosos usuários de quatro CCI existentes no Município de Cuiabá-MT, com um total de 1817 idosos cadastrados, sendo 1369 do sexo feminino e 448 do sexo masculino.

\subsubsection{Amostragem, critérios de inclusão e exclusão}

No trabalho de Rodrigues (2013) na determinação do tamanho da amostra foi utilizado um planejamento amostral do tipo probabilístico, considerando os métodos de amostragem aleatória simples e estratificada proporcional ao número e sexo de idosos em cada CCI. Assim, utilizando estes métodos de amostragem o tamanho da amostra aproximado na pesquisa foi de 317 idosos, considerando o tamanho da população dos quatro CCI e uma proporção de $50 \%(p=0,50)$, um erro de $5 \%(d=$ $0,05)$ e um nível de confiança de $95 \%(z=1,96)$, detalhes do planejamento amostral podem ser encontrados em Espinosa et al. (2015).

Para a condução do presente estudo utilizou-se o cri- 
tério de inclusão: idosos de ambos os sexos, com 60 anos ou mais de idade e que frequentaram o CCI nos últimos seis meses ou mais. Foram excluídos do estudo os indivíduos com incapacidades funcionais e cognitivas que os impossibilitaram de responder o questionário e que não responderam mais do que $20 \%$ do total de questões dos instrumentos WHOQOL-BREF e WHOQOL-OLD.

\subsubsection{Coleta de dados, questionário e instrumentos}

Conforme o projeto matricial, para a coleta dos dados foi aplicado um questionário estruturado que contém questões sobre as variáveis demográficas, socioeconômicas e de saúde, além dos instrumentos WHOQOL-BREF e WHOQOL-OLD, ambos validados no Brasil (FLECK et al., 2000; FLECK et al., 2006). O WHOQOL-BREF é constituído por quatro domínios: físico; psicológico; relações sociais e meio ambiente (FLECK et al., 2000). O WHOQOL-OLD é instrumento complementar do WHOQOL-BREF, utilizado exclusivamente para idosos, sendo composto por seis domínios: funcionamento dos sentidos, autonomia, atividades passadas, presentes e futuras, participação social, morte e morrer e intimidade (FLECK et al., 2006). A pesquisa foi realizada após o consentimento da gerência dos CCI e para entrevistar os idosos foi disponibilizado pela gerência dos serviços, um local reservado, e depois de identificado o idoso, o mesmo era convidado para participar do estudo. Maiores detalhes sobre os instrumentos e coleta dos dados podem ser encontrados no trabalho de Rodrigues (2013).

\subsubsection{Variáveis independentes e dependente}

Segundo o projeto matriz, as variáveis independentes foram classificadas em variáveis demográficas, socioeconômicas e de saúde, assim como os domínios do WHOQOL-BREF e WHOQOL-OLD.

A variável dependente principal foi a qualidade de vida geral ou global (QVG) do WHOQOL-BREF.

Observar que, cada domínio dos instrumentos utilizados é constituído por questões, cujas pontuações das respostas variam entre o valor 1 e 5 , sendo quanto mais próxima de 5 , melhor a qualidade de vida. Os escores finais de cada domínio são calculados por uma sintaxe, que considera as respostas de cada questão que compõe o domínio, resultando em escores finais numa escala de 4 a 20 , que podem ser transformados em escala de 0 a 100 que possibilita expressar o escore da escala em percentagem entre o valor mais baixo possível (0) e o mais alto possível (100). Basicamente, escores altos representam melhor qualidade de vida e escores baixos pior qualidade de vida (WHOQOL GROUP, 1998). E estas porcentagens também podem ser categorizadas utilizando a média ou a mediana como medidas de corte, dependendo da distribuição de probabilidade das porcentagens dos escores dos domínios serem simétricos ou assimétricos.

No presente trabalho foram utilizadas as porcentagens dos escores da qualidade de vida e também considerando os valores da qualidade de vida categorizados como menores que a mediana e maiores ou iguais a mediana, sendo estas categorias representativas de uma pior e melhor qualidade de vida, respectivamente. Assim, para avaliar os efeitos que as variáveis independentes exercem sobre a qualidade de vida, foram aplicados dois modelos estatísticos: modelo de regressão linear múltipla quantitativa e modelo de regressão de Poisson múltipla.

\section{Modelos de regressão, processamento e análise dos dados}

\subsubsection{Modelo de Regressão Linear Múltiplo Quanti- tativo}

O modelo de regressão linear múltipla pode ser escrito da seguinte maneira:

$$
y_{i}=a_{0}+a_{1} x_{1 i}+a_{2} x_{2 i}+\cdots+a_{k} x_{k}+\varepsilon_{i}
$$

no qual $x_{1 i}, x_{2 i}, \cdots, x_{k}$ são as variáveis independentes, para $i=1,2, \cdots, n$ e $k$ o número de estas variáveis independentes, $a_{0}, a_{1}, \cdots, a_{k}$ são os parâmetros do modelo dado pela expressão (1), denominados também de coeficientes de regressão e $\varepsilon_{i}$ são os erros aleatórios.

Os $\varepsilon_{i}$ são variáveis aleatórias com as seguintes suposições (DRAPER; SMITH, 1998):

A média dos $\varepsilon_{i}$ é zero e sua variância $\varphi^{2}$ é desconhecida e constante, para $1 \leq i \leq n$;

A distribuição dos $\varepsilon_{i}$ é normal, para $1 \leq i \leq n$.

Os $\varepsilon_{i}$ são não-correlacionados;

A segunda e terceira suposição implica que os erros aleatórios $\left(\varepsilon_{i}\right)$ são mutuamente independentes.

O modelo de regressão linear múltiplo da expressão (1) em notação matricial pode ser escrito da seguinte maneira:

$$
\widetilde{Y}=X \widetilde{A}+\widetilde{\varepsilon}
$$

na qual $\widetilde{Y}$ é um vetor $n \times 1$ das observações da variável dependenłe ou resposta, $X$ é uma matriz $n \times(k+1)$ formada pelas variáveis independentes mais uma constante, $\widetilde{A}$ é um vetor $(k+1) \times 1$ de parâmetros desconhecidos e $\widetilde{\mathcal{E}}$ é um vetor $n \times 1$ dos erros aleatórios, com as suposições 1, 2 e 3 apresentadas acima (DRAPER; SMITH, 1998).

Para estimar os parâmetros do modelo da expressão (2) é utilizado o método de mínimos quadrados e para verificar o cumprimento das suposições do modelo ajustado é realizada uma análise dos resíduos, usualmente utilizando métodos gráficos ad hoc. Na pratica, para verificar a primeira suposição, isto é, para verificar se a variância é constante, pode-se construir o gráfico de dispersão dos resíduos ou dos resíduos padronizados versus valores ajustados. No entanto, também podem ser realizados testes estatísticos para esta finalidade, por exemplo, utilizando o teste de Breusch-Pagan/Cook-Weisberg (COOK; WEISBERG, 1983). E a segunda suposição pode ser verificada construindo o gráfico de probabilidade normal dos resíduos, neste caso também podem ser utilizados testes estatísticos, tais como o teste de Shapiro-Wilk e Anderson-Darling, entre outros (DRAPER; SMITH, 1998; CHATTERJEE ; PRICE, 2006; KUTNER et al., 2005).

Após verificar que um modelo de regressão linear é adequado, o mesmo pode ser utilizado para realizar 
inferências sobre a variável resposta por meio dos seus parâmetros estimados. No entanto, os dados de qualidade de vida expresso em porcentagem, frequentemente não se adequam a um modelo linear. Nesta situação, nos estudos de cortes transversais ou longitudinais, uma alternativa é utilizar os dados dos escores da qualidade de vida categorizando-os como qualidade de vida menor que a mediana e maior ou igual que a mediana, considerando um modelo não linear, tal como o modelo de regressão de Poisson múltiplo (BARROS; HIRAKATA, 2003; LE, 2003; COUTINHO et al., 2008).

\subsubsection{Análise de prevalência}

Particularmente em estudos de corte transversal ou de prevalência com a variável dependente dicotômica, a associação entre esta variável e as variáveis explicativas ou fatores de exposição pode ser estimada por modelos de regressão logística, porém, quando os valores da variável dependente não são raros, ou quando é necessário ajustar para potenciais variáveis de confusão ou analisar a interação entre as variáveis, a razão de chances (odds ratio) não é uma boa estimativa para a razão de prevalência, pois em geral os parâmetros são subestimados ou superestimados. Uma alternativa bastante utilizada nesta situação é a utilização da razão de prevalência e do modelo de regressão de Poisson (KUTNER et al., 2005; BARROS; HIRAKATA, 2003; LE, 2003; COUTINHO et al., 2008).

A prevalência é definida como a frequência de casos existentes de uma dada doença em uma determinada população e em um dado momento. Assim, para se medir a prevalência, os indivíduos componentes de uma amostra são observados uma única vez.

Os dados obtidos por meio de um estudo de corte transversal usualmente são apresentados em uma tabela de contingencia formada por duas linhas e duas colunas, geralmente denominada de tabela $2 \times 2$, pela natureza dicotômica das variáveis de doença e de exposição, conforme ilustrado na Tabela 1. Convencionalmente, as colunas da tabela $2 \times 2$ representam a presença ou ausência de doença e as linhas a presença ou ausência de exposição.

Tabela 1 - Frequências de doença e exposição observadas em um estudo seccional

\begin{tabular}{lccc}
\hline \multirow{2}{*}{ Exposição } & \multicolumn{2}{c}{ Doença } & \multirow{2}{*}{ Total } \\
\cline { 2 - 3 } & Doentes & Não doentes & \\
\hline Expostos & $\mathrm{a}$ & $\mathrm{b}$ & $\mathrm{a}+\mathrm{b}$ \\
Não expostos & $\mathrm{c}$ & $\mathrm{d}$ & $\mathrm{c}+\mathrm{d}$ \\
\hline Total & $\mathrm{a}+\mathrm{c}$ & $\mathrm{b}+\mathrm{d}$ & $\mathrm{n}$ \\
\hline
\end{tabular}

Em estudos de prevalência, para mensurar a associação entre uma variável dependente e variáveis independentes é utilizada a razão de prevalência $(R P)$ ou razão de prevalência bruta $\left(R P_{b}\right)$ dada por (3):

$$
\boldsymbol{R}_{b}=\frac{a(a+b)}{c(c+d)}=\frac{a(c+d)}{c(a+b)}
$$

a qual estima quantas vezes mais os expostos estão doentes quando comparados aos não expostos na época do estudo seccional.

Um intervalo de confiança para a razão de prevalência é dado por (4):

na qual o desvio padrão da razão de prevalência $(D P R P)$ é dada pela seguinte expressão:

$$
\left[\boldsymbol{R} \times \exp \left(-z_{1-\alpha / 2} \times D P R P\right) ; \boldsymbol{R} \times \exp \left(z_{1-\alpha / 2} \times D P R P\right]\right.
$$

2.2.3 Modelo de Regressão de Poisson múltiplo

$$
D P R P=\left(\frac{b}{a(a+b)}+\frac{d}{c(c+d)}\right)^{1 / 2}=\sqrt{\frac{b}{a(a+b)}+\frac{d}{c(c+d)}}
$$

O modelo de regressão de Poisson pode ser utilizado em estudos epidemiológicos longitudinais, nos quais a resposta é o número de ocorrências de um evento em um determinado período de tempo. Para estudo de corte transversal ou de prevalência, nos quais todos os indivíduos têm tempo de seguimento igual, a regressão de Poisson pode ser utilizada com o valor de tempo sob risco igual a 1 para cada indivíduo. Neste caso é possível atribuir o valor unitário ao tempo de seguimento de cada participante como estratégia para obtenção da estimativa por ponto da razão de prevalência, pois não há seguimento real dos participantes nesse tipo de estudo epidemiológico. Porém quando a regressão de Poisson é aplicada em dados binários, o erro para o risco relativo é superestimado, uma vez que a variância da distribuição de Poisson aumenta progressivamente, enquanto a variância da distribuição binomial atinge seu valor máximo ao ter uma prevalência de 0,5.

Assim, quando é atribuído um período de risco constante a todos as unidades, a razão de risco estimada é igual à razão de prevalência $(R P)$. No entanto como a variância da distribuição de Poisson nesta situação aumenta, os coeficientes tendem a serem superestimadas, resultando em intervalos de confiança maiores (BARROS; HIRAKATA, 2003). Para contornar essa situação pode ser utilizado o método de variância robusta (COUTINHO et al., 2008; LIN; WEI, 1989), pois neste caso os valores ajustados pelo modelo de regressão de Cox coincidem com o modelo de regressão de Poisson e os parâmetros estimados são os mesmos (COLÓSIMO; GIOLO, 2006; BARROS; HIRAKATA, 2003; COUTINHO et al., 2008).

Assim, o modelo de regressão de Poisson múltiplo (6) pode ser escrito da seguinte maneira:

$$
E\left(Y_{i}\right)=\exp (\tilde{X} \widetilde{\beta})
$$

para $i=1,2, \ldots, n$, na qual $\exp (\widetilde{X} \widetilde{\beta})$ é denominado de risco relativo.

Os coeficientes de regressão do modelo (6), $\beta_{i}$ são estimados pelo método da máxima verossimilhança. Observar que para obter estes estimadores são necessários métodos numéricos, geralmente utilizando os pacotes estatísticos, tais como o SPSS, Stata, entre outros (KUTNER et al., 2005).

Utilizando os valores dos parâmetros estimados por métodos numéricos pode ser obtida uma estimativa 
pontual para a razão de prevalência ajustada dada por (7):

$$
\boldsymbol{R}_{a}=\exp \left(B_{j}\right)=\exp \left(\hat{\beta}_{j}\right)
$$

para $j=1,2, \ldots, k$ na qual o valor de $\exp \left(B_{j}\right)$ será a razão de prevalência ajustada, tanto para variáveis categóricas como contínuas (LE, 2003).

A razão de prevalência ajustada $\left(\boldsymbol{R}_{a}\right)$ pode ser qualquer número não negativo e é uma medida que visa mensurar a associação entre uma variável resposta e uma variável independente. Geralmente uma variável de ocorrência de uma doença ou desfecho e um fator ou variável de exposição, representando cada uma dois eventos, embora a variável independente possa ser utilizada de maneira quantitativa. Assim, a razão de prevalência mede a força da associação entre duas variáveis, isto é, quantas vezes a ocorrência da doença é maior no evento de exposição ou grupo de expostos em relação ao evento de não exposição ou grupo de não expostos.

A $R_{a}$ do evento ou categoria de base é sempre igual a $1\left(\boldsymbol{R}_{a}=1\right)$ e serve como a linha base para a comparação das categorias das variáveis independentes. Assim, se o valor da $\boldsymbol{R}_{a}$ da categoria ou das categorias de não referencia for igual ou aproximadamente igual a um ( $\boldsymbol{R}_{a}=1$ ), isso indica que não existe associação entre a resposta e a variável explicativa. Se $R_{a}>1$, a razão de prevalência ajustada da categoria de interesse é maior e caracteriza um fator de risco (frequência de doença maior nos expostos em relação aos não expostos). Se $\boldsymbol{R}_{a}<1$, a possibilidade de ocorrência da categoria de interesse é menor e caracteriza um fator de proteção (frequência de doença menor nos expostos em relação aos não expostos).

Estimado o modelo de regressão de Poisson múltiplo é preciso verificar sua adequação para realizar inferências sobre os parâmetros de interesse. Isto pode ser realizado fazendo um teste de hipóteses geral entre a associação da variável dependente e as variáveis independentes, pelo método da razão de verossimilhança ou por outros testes, os quais podem ser encontrados em Agresti (2007) e Kutner et al. (2005), entre outros. Após verificar que o modelo é adequado, podem ser realizados testes de hipóteses sobre os parâmetros com um determinado nível de significância, usualmente considerando valores de $\mathrm{p}$ menor que 0,05 , para saber se um fator de risco ou de proteção é estatisticamente significativo. No entanto, para realizar inferências sobre os parâmetros, também podem ser utilizados intervalos de confiança, os quais fornecem informações recíprocas às dos testes de hipóteses, dada uma confiabilidade complementar ao nível de significância nominal dos testes (LE, 2003; AGRESTI, 2007).

\subsubsection{Processamento e análises dos dados}

No presente trabalho, os dados foram processados e analisados nos pacotes estatísticos MINITAB versão 17 (Minitab 17 Statistical Software 2010) e SPSS versão 20 (IBM Corp. Released 2011). Antes da análise categórica foi verificada a distribuição dos escores dos domínios da qualidade de vida expressos em porcentagem utilizando os testes de Shapiro e Anderson (DRAPER; SMITH, 1998). Após verificar que todos os escores dos domínios não possuíam distribuição simétrica, foram feitas as categorizações dos domínios de ambos os instrumentos e a seguir realizadas as associações entre a QVG e as variáveis independentes em estudo. Para realizar estas associações, primeiro foi utilizada uma análise categórica bivariada por meio da razão de prevalência bruta $\left(R P_{b}\right)$ com seus respectivos intervalos de confiança e valores de $\mathrm{p}$, considerando o teste qui quadrado de Pearson, e a seguir, as variáveis que apresentaram associação com níveis exatos de significância ( $p$-valores) menores que $0,20(\mathrm{p}<0,20)$ foram selecionadas para compor o modelo de regressão de Poisson múltiplo com variância robusta, permanecendo no modelo final somente aquelas que apresentaram $\mathrm{p}$-valores menores que $0,05(p<0,05)$. A adequação deste modelo ajustado foi verificada pelo teste da razão de verossimilhança (LE, 2003; BARROS e HIRAKATA, 2003; COUTINHO et al, 2008). Em todas as inferências foram consideradas intervalos de confiança de $95 \%$ e nível de significância de $5 \%$.

\section{Considerações éticas}

De acordo com o projeto matriz, o mesmo foi submetido ao Comitê de Ética e Pesquisa envolvendo seres humanos do Hospital Universitário Júlio Muller, com aprovação sob o número de protocolo 36090/CEP-HUJM/2012 e CAAE № 01989212.7.0000.5541. Todos os participantes assinaram o Termo de Consentimento Livre e Esclarecido, de acordo com os requisitos da resolução 466/2012 do Conselho Nacional de Saúde.

\section{Resultados e discussões}

Inicialmente, foi realizada uma análise descritiva utilizando a mediana, média e desvio padrão dos escores de qualidade de vida dos domínios do WHOQOL-BREF e WHOQOL-OLD dos idosos dos CCI, conforme apresentado na Tabela 2. Nesta tabela, observa-se que o domínio do WHOQOL-BREF com maior escore mediano foi o psicológico $(75,00 \%)$ e com menor escore mediano foi o meio ambiente $(59,38 \%)$. O grupo de idosos apresentou uma qualidade de vida global mediana de $62,50 \%$. Enquanto que os domínios do WHOQOL-OLD com maiores escores medianos foram morte e morrer $(87,50 \%)$ e participação social $(68,75 \%)$ e com menor escore mediano foi funcionamento dos sentidos (56,25\%).

A seguir foi verificada a distribuição simétrica dos escores dos domínios pelos testes de Shapiro e Anderson, identificando que nenhum dos escores dos domínios apresentou um comportamento linear, mesmo considerando transformações aos dados, como o método de Box e Cox. Assim, a aplicação do modelo linear seria inadequado para analisar os escores dos domínios na escala percentual, pois o mesmo levaria a inferências equivocadas (DRAPER; SMITH, 1998; CHATTERJEE ; PRICE, 2006). Embora os pacotes estatísticos realizem os cálculos e apresentem os coeficientes, intervalos de confiança de $95 \%$ e p-valores dos parâmetros estimados das variáveis independentes do modelo inadequado, obtidos, 
Tabela 2 - Mediana, média e desvio padrão dos escores de qualidade de vida dos idosos dos CCl, segundo WHOQOL-BREF e WHOQOL-OLD, Cuiabá-MT, 2012

\begin{tabular}{|c|c|c|c|c|}
\hline Escores de QV & Mediana & Média & $\mathrm{DP}$ & $\mathrm{CV}$ \\
\hline \multicolumn{5}{|l|}{ WHOQOL-BREF } \\
\hline Físico & 71,43 & 69,33 & 10,88 & 15.69 \\
\hline Psicológico & 75,00 & 72,45 & 7,69 & $\begin{array}{l}10,04 \\
10,61\end{array}$ \\
\hline Relações sociais & 66,67 & 70,19 & 11,24 & $\begin{array}{l}10,61 \\
1601\end{array}$ \\
\hline Meio ambiente & 59,38 & 57,47 & 7,37 & $\begin{array}{l}10, \cup 1 \\
12,82\end{array}$ \\
\hline QV & 62,50 & 65,97 & 14,27 & $\begin{array}{l}12,02 \\
21,63\end{array}$ \\
\hline \multicolumn{5}{|l|}{ WHOQOL-OLD } \\
\hline Funcionamento dos sentidos & 56,25 & 56,86 & 6,21 & 10,92 \\
\hline Autonomia & 62,50 & 61,14 & 11,65 & $\begin{array}{l}10,72 \\
19,05\end{array}$ \\
\hline Atividades passadas, presentes e futuras & 62,50 & 61,26 & 11,84 & 19,33 \\
\hline Participação social & 68,75 & 69,07 & 7,42 & 10,74 \\
\hline Morte e morrer & 87,50 & 81,74 & 22,11 & 27,05 \\
\hline Intimidade & 62,50 & 59,25 & 18,48 & 31.19 \\
\hline
\end{tabular}

DP: Desvio Padrão; CV: coeficiente de variação relativo

por exemplo, pelo método passo a passo (stepwise), mesmo que estes estimadores mostrem "significância estatística”, serão inadequados para fazer qualquer inferência. Conforme mostrado na Tabela 3, os pacotes estatísticos calcularam os valores utilizados nas inferências e ainda estes valores apresentaram "significância estatística", mesmo o modelo tendo sido inadequadamente aplicado. No entanto, neste caso esta significância não deve ser considerada para realizar conclusões, pois o modelo não cumpre os supostos básicos de um modelo de regressão linear quantitativo.

Tabela 3 - Coeficientes de regressão das variáveis independentes, DP, IC e p-valor, para os dados do modelo da expressão (2)

\begin{tabular}{c|c|c|c|c}
\hline Variável & Coeficiente & DP & IC 95\% & p-valor \\
\hline Constante & 6,0500 & 7,9500 & $(-9,5900 ; 21,6900)$ & 0,4470 \\
\hline Físico & 0,3955 & 0,0762 & $(0,2456 ; 0,5454)$ & $<0,001$ \\
\hline Psicológico & 0,2400 & 0,1030 & $(0,0360 ; 0,4430)$ & 0,021 \\
\hline $\begin{array}{c}\text { Meio } \\
\text { ambiente }\end{array}$ & 0,3694 & 0,0961 & $(-0,1876 ;-0,0054)$ & $<0,001$ \\
\hline $\begin{array}{c}\text { Realiza } \\
\text { caminhada }\end{array}$ & $-2,8500$ & 1,3800 & $(-5,5600 ;-0,1400)$ & 0,039 \\
\hline $\begin{array}{c}\text { Percepção } \\
\text { de saúde }\end{array}$ & $-4,7000$ & 1,9600 & $(-8,5600 ;-0,8400)$ & 0,017 \\
\hline $\begin{array}{c}\text { Problemas } \\
\text { de saúde }\end{array}$ & $-4,1400$ & 1,8200 & $(-7,7100 ;-0,5700)$ & 0,023 \\
\hline \multicolumn{5}{c}{$\mathrm{R} 2=0,3942 \mathrm{n}=317 \mathrm{~s}=11,2105 \mathrm{p}$-valor < 0,001 } \\
\hline
\end{tabular}

Observar que o modelo apresentado na Tabela 3, embora os coeficientes apresentem "significância estatística” o mesmo não é adequado, conforme evidenciado na Figura 1, uma vez que a variância dos resíduos não é constante, embora o gráfico dos resíduos apresente distribuição normal.

Considerando que o modelo da Tabela 3 não foi adequado, foram tentadas diversas transformações pelo método de Box-Cox (DRAPER; SMITH, 1998), mas não foi possível obter uma adequação do modelo linear. Desta forma a variável resposta foi categorizada, con-
Figura 1 - Gráfico dos resíduos versus valores ajustados, para os dados da Tabela 3.

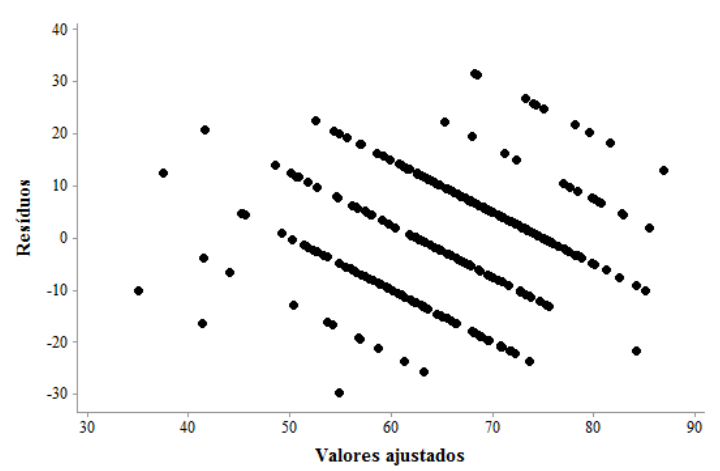

Figura 2 - Gráfico de probabilidade normal dos resíduos, para os dados da Tabela 3.

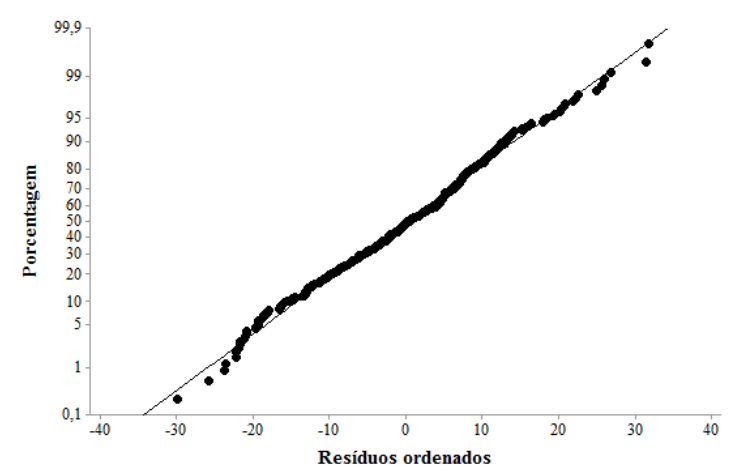

siderando o ponto de corte como melhor qualidade de vida, os indivíduos com escores maiores ou iguais ao valor da mediana e como pior qualidade de vida os que apresentaram escores menores a este valor. Com esta categorização, primeiro foi feita uma análise categórica utilizando a razão de prevalência bruta apresentada nas Tabelas 4, 5 e 6 .

$\mathrm{Na}$ Tabela 4 são apresentadas as associações entre os escores do domínio da QVG menor que a mediana e 
variáveis socioeconômicas dos idosos dos CCI com suas respectivas razões de prevalências brutas, intervalos de confiança de $95 \%$ e valores de p. Nesta análise bivariada, não foram verificadas associações estatisticamente significantes com variáveis socioeconômicas.

As associações entre os escores do domínio da QVG menor que a mediana e as variáveis aspectos de saúde dos idosos dos CCI são apresentados na tabela 5, com suas respectivas razões de prevalências brutas, intervalos de confiança de $95 \%$ e p-valores. Nesta análise bivariada, para as variáveis referentes aos aspectos de saúde, apenas a variável serviço de saúde utilizado não foi estatisticamente significativa.

A percepção de saúde estar doente evidenciou forte associação com a QVG menor que a mediana $\left(R P_{b}=2,81\right)$, verificando que esta percepção pode influenciar na QVG do idoso. Este resultado confirma os encontrados no estudo realizado com idosos comunitários da macrorregião do Triângulo do Sul, Minas Gerais, Brasil, realizado por Paiva et al. (2016).

A QVG menor que a mediana associou-se a não realizar caminhada de maneira significativa $\left(R P_{b}=2,01\right)$. Este resultado corrobora com o encontrado no estudo de Pagotto et al. (2011), os quais verificaram que não praticar atividade física apresentou-se associado à auto avaliação do estado de saúde ruim e consequentemente pior percepção de qualidade de vida. Confirmando os resultados encontrados na presente pesquisa, no qual não realizar caminhada evidenciou ser um fator de risco na QVG menor que a mediana.

Os problemas de saúde também mostraram associação de risco, altamente significativa $(\mathrm{p}<0,001)$, com a QVG menor que a mediana, indicando que um indivíduo idoso possui 4 vezes mais chances de ter uma qualidade de vida inferior do que idosos sem problemas de saúde. Evidenciando que os idosos com problemas de saúde apresentaram menores escores neste domínio do que os idosos sem problemas de saúde, isto é, idosos com enfermidades percebem pior sua qualidade de vida e estes resultados confirmam os encontrados em outros estudos (VAGETTI et al., 2013; FORTIN et al., 2004).

Outra associação entre QVG menor que a mediana foi com o uso de medicação. O uso de medicação por idosos pode afetar a qualidade de vida geral dos mesmos. Segundo Paiva et al (2016), as reações adversas à medicação constituem um problema importante na relação medico-paciente, pois estas reações podem influenciar na perda da confiança do paciente com seu médico, e em decorrência afetar a QVG.

A QVG menor que a mediana apresentou apenas uma

Tabela 4 - Associação entre QVG menor que a mediana e variáveis socioeconômicas dos idosos dos CCl, com suas respectivas razões de prevalências brutas, intervalo de confiança de 95\% e valores de p, Cuiabá-MT, 2012.

\begin{tabular}{|c|c|c|c|c|c|}
\hline Características & $\begin{array}{l}\text { QVG < } \\
\text { mediana }\end{array}$ & $\begin{array}{l}\mathrm{QVG} \geq \\
\text { mediana }\end{array}$ & $\mathrm{RP}_{\mathrm{b}}$ & IC 95\% & $\mathrm{p}$-valor \\
\hline \multicolumn{6}{|l|}{ Sexo } \\
\hline Feminino & 68 & 171 & 1,00 & $\begin{array}{c}(0,00 ; 1,47) \\
-\end{array}$ & - \\
\hline \multicolumn{6}{|l|}{ Faixa etária (em anos) } \\
\hline 60 a 69 anos & 44 & 107 & 1,00 & - & - \\
\hline 70 a 79 anos & 38 & 98 & 0,96 & $(0,66 ; 1,38)$ & 0,823 \\
\hline 80 anos e mais & 8 & & 0,92 & $(0,48 ; 1,74)$ & 0,785 \\
\hline \multicolumn{6}{|l|}{ Estado Conjugal } \\
\hline Sem companheiro & 59 & 156 & 0,90 & $(0,63 ; 1,30)$ & 0,586 \\
\hline \multicolumn{6}{|l|}{ Escolaridade (em anos) } \\
\hline Analfabeto & 25 & 60 & 1.21 & $(0.64 \cdot 2.27)$ & 0555 \\
\hline 1 a 4 anos & 43 & 92 & 1,31 & $(0,72 ; 2,36)$ & 0,362 \\
\hline 5 a 8 anos & 12 & 44 & 0,88 & $(0,42 ; 1,83)$ & 0,731 \\
\hline 9 anos ou mais & & - \\
\hline $\begin{array}{l}\text { Rellgao } \\
\text { Outras }\end{array}$ & & 52 & & $(0,82 ; 1,76)$ & 0.362 \\
\hline Católica & 65 & 175 & 1,00 & $\begin{array}{c}- \\
-10,0<10\end{array}$ & - \\
\hline \multicolumn{6}{|l|}{ Renda individual (salário mínimo)** } \\
\hline Nenhuma/O<SM 1 & 68 & 164 & 1,13 & $(0,75 ; 1,71)$ & 0,549 \\
\hline$>1 \mathrm{SM}$ & 22 & 63 & 1,00 & & \\
\hline \multicolumn{6}{|l|}{ Classe econômica } \\
\hline$C / D$ & 85 & 216 & 0,90 & $(0,43 ; 1,91)$ & $0,780^{*}$ \\
\hline B & 5 & 11 & 1,00 & - & \\
\hline \multicolumn{6}{|l|}{ Atividade na comunidade } \\
\hline Não & 81 & 187 & 1,65 & $(0,89 ; 3,05)$ & 0,091 \\
\hline \multirow{2}{*}{\multicolumn{6}{|c|}{ Percepção de apoio social }} \\
\hline & & & & & \\
\hline Não & 25 & 68 & 0,93 & $(0,62 ; 1,37)$ & 0,701 \\
\hline Sim & 65 & 159 & 1,00 & (5) & \\
\hline
\end{tabular}

**: O valor do salário mínimo era de R\$ 622,00. *: Teste exato de Fisher. 
Tabela 5 - Associação entre a QVG menor que a mediana e variáveis aspectos de saúde dos idosos dos CCl, com suas respectivas razões de prevalências brutas, intervalo de confiança de 95\% e valores de p, Cuiabá-MT, 2012.

\begin{tabular}{|c|c|c|c|c|c|}
\hline Características & $\begin{array}{l}\text { QVG < } \\
\text { mediana }\end{array}$ & $\begin{array}{l}\text { QVG } \geq \\
\text { mediana }\end{array}$ & $\mathrm{RP}_{\mathrm{b}}$ & IC 95\% & p-valor \\
\hline \multicolumn{6}{|c|}{ Percepção de saúde } \\
\hline Doente & 31 & 19 & 2,81 & $(2,05 ; 3,84)$ & $<0,001$ \\
\hline Saudável & 59 & 208 & 1,00 & - & - \\
\hline \multicolumn{6}{|c|}{ Realiza caminhada } \\
\hline Não & 72 & 139 & 2,01 & $(1,27 ; 3,19)$ & 0,001 \\
\hline Sim & 18 & 88 & 1,00 & - & \\
\hline \multicolumn{6}{|c|}{ Problemas de saúde } \\
\hline $\operatorname{Sim}$ & 86 & 180 & 4,12 & $(1,58 ; 10,73)$ & $<0,001$ \\
\hline Não & 4 & 47 & 1,00 & - & - \\
\hline \multicolumn{6}{|c|}{ Uso de medicação } \\
\hline $\operatorname{sim}$ & 82 & 176 & 2,34 & $(1,20 ; 4,57)$ & 0,005 \\
\hline Não & 8 & 51 & 1,00 & - & - \\
\hline \multicolumn{6}{|c|}{ Serviço de saúde utilizado } \\
\hline Público & 70 & 160 & 1,32 & $(0,86 ; 2,04)$ & 0,189 \\
\hline Particular & 20 & 67 & 1,00 & - & - \\
\hline
\end{tabular}

tendência fraca de associação com o serviço de saúde público utilizado, embora sem significância estatística $(\mathrm{p}=0,189)$, isto pode evidenciar que os idosos que não possuíam um plano de saúde particular podiam ter tido maiores problemas de saúde, o qual pode ter influenciado na pior QVG observada nos idosos dos CCI estudados no presente estudo (PEREIRA et al., 2011).

As associações entre os escores do domínio da QVG menor que a mediana e os escores dos domínios do WHOQOL-BREF e WHOQOL-OLD menor que a mediana dos idosos dos CCI são apresentadas na Tabela 6. Nesta tabela, observa-se que as associações com os domínios relações sociais, funcionamento dos sentidos e morte e morrer não foram estatisticamente significativas.

No presente estudo, encontrou-se que as associações entre os escores do domínio QVG menor que a mediana e os escores dos domínios do WHOQOL-BREF menores que a mediana foram com os domínios físico, psicológico e meio ambiente $\left(R P_{b}=2,95, R P_{a}=2,77\right.$ e $\left.R P_{a}=1,65\right)$, respectivamente.

A associação mais forte dos escores do domínio físico com a QVG em ambos os casos menor que a mediana dos idosos considerados, destaca a importância da necessidade das atividades físicas na QVG destes indivíduos. Este resultado evidencia o papel fundamental destas atividades, para uma melhor promoção de QVG dos idosos. Segundo Vagetti et al. (2013), a realização diária de atividades físicas pode contribuir para a melhoria de uma boa percepção de saúde e bem-estar desta população e consequentemente uma melhor QVG.

A associação da QVG menor que a mediana com os escores do domínio psicológico, mostrou que as mudanças e alterações nos idosos, tais como físicas, econômicas e sociais podem afetar a QVG desses indivíduos. Assim, segundo Vagetti et al. (2013) e Pereira et al. (2006) é necessário considerar estas alterações psicológicas para reduzir os efeitos dessas transformações nas distintas etapas do envelhecimento. Estes autores sugerem a participação destes indivíduos em programas de atividades físicas e sociais, visando melhores condições físicas e de saúde e também possibilitar novos relacionamentos afetivos e sociais, em decorrência de uma melhor QVG.

Outra associação de risco entre QVG menor que a mediana foi com o domínio meio ambiente. Este resultado pode ter associação com a baixa renda dos idosos, que por sua vez, poderá contribuir com condições escassas de segurança e proteção, dificuldades ao acesso dos serviços de saúde, limitações de recreação e lazer, entre outros (FLECK et al., 2000). Estes fatores mensurados por este domínio podem reduzir a qualidade de vida do idoso.

Ao analisar a contribuição dos diferentes escores dos domínios do WHOQOL-OLD, verificou-se que as associações entre estes domínios com os escores do domínio da QVG menor que a mediana, foram estatisticamente significativos para os domínios autonomia, atividades passadas, presentes e futuras, participação social e intimidade.

A associação da QVG menor que a mediana com os escores do domínio autonomia dos idosos abaixo da mediana, pode estar relacionados à menor liberdade para tomar suas decisões à medida que aumenta a idade destes indivíduos, e a impossibilidade de não poder fazer aquilo que gostariam de fazer devido às suas limitações ou falta 
Tabela 6 - Associação entre QVG menor que a mediana e escores dos domínios do WHOQOL-BREF e WHOQOL-OLD menor que a mediana dos idosos dos $\mathrm{CCl}$, com suas respectivas razões de prevalências brutas, intervalo de confiança de $95 \%$ e p-valores, Cuiabá-MT, 2012.

\begin{tabular}{|c|c|c|c|c|c|}
\hline Características & $\begin{array}{l}\text { QVG < } \\
\text { mediana }\end{array}$ & $\begin{array}{l}\mathrm{QVG} \geq \\
\text { mediana }\end{array}$ & $\mathrm{RP}_{\mathrm{b}}$ & IC 95\% & p-valor \\
\hline \multicolumn{6}{|l|}{ WHOQOL-BREF } \\
\hline \multicolumn{6}{|l|}{ Físico } \\
\hline$<$ mediana & 73 & 115 & 2,95 & $(1,83 ; 4,75)$ & $<0,001$ \\
\hline zmediana & 17 & 112 & 1,00 & - & - \\
\hline \multicolumn{6}{|l|}{ Psicológico } \\
\hline$<$ mediana & 66 & 92 & 2,77 & $(1,83 ; 4,18)$ & $<0,001$ \\
\hline zmediana & 24 & 135 & 1,00 & - & - \\
\hline \multicolumn{6}{|l|}{ Relações Sociais } \\
\hline$<$ mediana & 20 & 33 & 1,42 & $(0,95 ; 2,12)$ & 0,098 \\
\hline zmediana & 70 & 194 & 1,00 & - & - \\
\hline \multicolumn{6}{|l|}{ Meio Ambiente } \\
\hline$<$ mediana & 69 & 142 & 1,65 & $(1,08 ; 2,54)$ & 0,016 \\
\hline zmediana & 21 & 85 & 1,00 & - & - \\
\hline \multicolumn{6}{|l|}{ WHOQOL-OLD } \\
\hline \multicolumn{6}{|c|}{ Funcionamento dos sentidos } \\
\hline$<$ mediana & 16 & 62 & 0,66 & $(0,41 ; 1,07)$ & 0,076 \\
\hline zmediana & 74 & 165 & 1,00 & - & - \\
\hline \multicolumn{6}{|l|}{ Autonomia } \\
\hline$<$ mediana & 53 & 85 & 1,86 & $(1,30 ; 2,65)$ & 0,001 \\
\hline 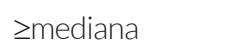 & 37 & 142 & 1,00 & - & - \\
\hline \multicolumn{6}{|c|}{$\begin{array}{l}\text { Atividades passadas, presentes e } \\
\text { futuras }\end{array}$} \\
\hline$<$ mediana & 56 & 84 & 2,08 & $(1,45 ; 3,00)$ & $<0,001$ \\
\hline zmediana & 34 & 143 & 1,00 & - & - \\
\hline \multicolumn{6}{|c|}{ Participação Social } \\
\hline$<$ mediana & 41 & 50 & 2,08 & $(1,49 ; 2,91)$ & $<0,001$ \\
\hline$\geq$ mediana & 49 & 177 & 1,00 & - & - \\
\hline \multicolumn{6}{|l|}{ Morte e morrer } \\
\hline$<$ mediana & 50 & 106 & 1,29 & $(0,91 ; 1,84)$ & 0,155 \\
\hline zmediana & 40 & 121 & 1,00 & - & - \\
\hline \multicolumn{6}{|l|}{ Intimidade } \\
\hline$<$ mediana & 47 & 90 & 1,44 & $(1,01 ; 2,04)$ & 0,042 \\
\hline$\geq$ mediana & 43 & 137 & 1,00 & - & - \\
\hline
\end{tabular}


Tabela 7 - Razão de prevalência ajustada pelo modelo de regressão de Poisson entre a QVG menor que a mediana e variáveis de saúde e os escores dos domínios do WHOQOL-BREF e WHOQOL-OLD menor que a mediana dos idosos dos CCI com seus respectivos intervalos de confiança de 95\% e p-valores, Cuiabá-MT, 2012.

\begin{tabular}{|c|c|c|c|}
\hline Características & $\mathrm{RPa}$ & IC 95\% & $\mathrm{p}$-valor \\
\hline \multicolumn{4}{|c|}{ Percepção de saúde } \\
\hline Doente & 1,75 & $(1,26 ; 2,42)$ & 0,001 \\
\hline Saudável & 1,00 & - & - \\
\hline \multicolumn{4}{|c|}{ Realiza caminhada } \\
\hline Não & 1,70 & $(1,10 ; 2,61)$ & 0,017 \\
\hline Sim & 1,00 & - & - \\
\hline \multicolumn{4}{|l|}{ Domínio Físico } \\
\hline$<$ mediana & 1,82 & $(1,10 ; 3,00)$ & 0,020 \\
\hline$\geq$ mediana & 1,00 & - & - \\
\hline \multicolumn{4}{|c|}{ Domínio Psicológico } \\
\hline$<$ mediana & 2,00 & $(1,30 ; 3,05)$ & 0,001 \\
\hline$\geq$ mediana & 1,00 & - & - \\
\hline
\end{tabular}

de independência parcial ou total. Segundo estudo realizado com idosos no Município de João Pessoa - PB, por Dias et al. (2012), o processo de envelhecimento reduz o desempenho motor e cognitivo satisfatório, necessário para a realização das atividades diárias. Assim, segundo estes autores são fundamentais o incremento de estratégias de prevenção e promoção de saúde que o auxiliem no envelhecimento com independência e autonomia, o que poderá melhorar a QVG nos idosos.

Outra associação entre QVG menor que a mediana foi com os escores do domínio atividades passadas, presentes e futuras abaixo da mediana, este resultado pode evidenciar que os idosos não estavam satisfeitos com suas conquistas em sua vida e possuíam poucas perspectivas com seus futuros, e consequentemente isto pode ter influenciado na QVG inferior a mediana.

A associação entre os escores do domínio participação social abaixo da mediana com os escores do domínio QVG menor que a mediana, pode estar relacionado com a insatisfação do nível de atividade cotidiana, com a utilização do tempo e com as oportunidades para participar em atividades da comunidade. Segundo Tavares et al. (2016), os idosos devem ser envolvidos em atividades sociais, pois as mesmas podem oferecer-lhes benefícios físicos, funcionais e cognitivos. Além disso, estas atividades ampliam as trocas de experiências, os fazem sentirem-se úteis e pertencentes à sociedade, o que pode contribuir com uma melhor QVG destes indivíduos.

A associação da QVG menor que a mediana com os escores do domínio intimidade dos idosos abaixo da mediana, pode estar relacionada com a insatisfação dos idosos de amar e serem amados e de um sentimento de companheirismo em suas vidas. Portanto, Tavares et al. (2016) sugere que deveriam ser realizadas atividades de capacitação destes indivíduos, sobre relações intimas conjuntamente com seus familiares, o que poderia melhorar a QVG dos idosos dos CCI.

Na Tabela 7 é apresentado o modelo final da análise de regressão de Poisson múltipla, com as variáveis que foram significativas ao nível de $5 \%$, com seus respectivos intervalos de confiança de $95 \%$ e valores de p. Observar que o modelo de regressão de Poisson múltiplo foi globalmente significativo pelo teste da razão de verossimilhança $(p<0,001)$ e que no mesmo foram analisadas todas as variáveis com valores de p inferiores a 0,20 na análise bivariada apresentadas nas Tabelas 4,5 e 6 .

$\mathrm{Na}$ análise de regressão de Poisson múltipla entre a QVG menor que a mediana e as variáveis sobre os aspectos de saúde e os escores dos domínios do WHOQOL-BREF e WHOQOL-OLD menor que a mediana, mostraram associações estatisticamente significativas as variáveis dos aspectos de saúde: percepção de saúde e realiza caminhada e os domínios físicos e psicológicos do WHOQOL-BREF.

\section{Conclusões}

Segundo WHOQOL-BREF e WHOQOL-OLD, o menor escore mediano de qualidade de vida foi o domínio meio ambiente e o funcionamento dos sentidos, respectivamente. Estes escores indicam que as condições limitadas de segurança e proteção e as dificuldades ao acesso dos serviços de saúde e das habilidades sensoriais afetam a percepção subjetiva dos idosos estudados, consequentemente uma pior QVG. No entanto, foi verificado que os escores medianos dos domínios em porcentagem foram superiores a $50 \%$, o que pode evidenciar que a qualidade de vida dos idosos em estudo foi satisfatória.

Em muitos trabalhos de qualidade de vida geralmente é aplicado o modelo de regressão linear múltiplo quantitativo, no entanto os escores desta variável frequentemente não seguem um comportamento linear, mesmo com uma transformação dos dados da variável resposta, o qual foi evidenciado no presente estudo. Nesta situação, a utilização deste modelo levaria a conclusões equivocadas. Assim, é preciso ter cuidado na aplicação dos modelos de regressão, sempre verificando sua adequação, para 
reduzir a possibilidade de inferências inadequadas.

Uma alternativa verificada neste estudo foi a aplicação da razão de prevalência bruta e do modelo de regressão de Poisson múltiplo com os dados dos escores categorizados, uma vez que este modelo não é linear e também considerando que o estudo é do tipo transversal. No entanto, também é preciso verificar adequação deste modelo pelo método da razão da verossimilhança ou por outros testes.

A razão de prevalência bruta e o modelo de regressão de Poisson múltiplo indicaram que na qualidade de vida global abaixo da mediana, as variáveis e escores dos domínios do WHOQOL-BREF e WHOQOL-OLD menor que a mediana, que individualmente e conjuntamente apresentaram maiores riscos foram: problemas de saúde, uso de medicação, os escores dos domínios atividades passadas, presentes e futuras e participação social menor que a mediana e a percepção de saúde doente, não realizar caminhada e os escores dos domínios físico, psicológico, menor que a mediana. Variáveis e domínios de qualidade de vida que exerceram efeito negativo na QVG dos idosos, sendo assim, as mesmas devem ser consideradas nas ações de politicas públicas, visando uma melhoria sobre a qualidade de vida de essa população.

Finalmente pode ser concluído que dos modelos considerados, apenas o modelo de regressão de Poisson múltiplo foi adequado aos dados de qualidade de vida global dos idosos dos CCI do presente estudo.

\section{Agradecimentos}

A todos os profissionais dos Centros de Convivência para Idosos (CCI) no Município de Cuiabá-MT, à Secretaria Municipal de Saúde e à Secretaria Municipal de Assistência Social e Desenvolvimento Humano de Mato Grosso pela autorização para realização desta pesquisa e ao Conselho Nacional de Desenvolvimento Cientifico e Tecnológico ( $\mathrm{CNPq}$ ), pelo apoio financeiro por meio de Bolsa de Estudo da pesquisadora Dayane de Carvalho Rodrigues.

\section{Referências}

AGRESTI, A. An introduction to categorical data analysis. Second Edition. Published by John Wiley \& Sons, Inc., Hoboken, New Jersey. Published simultaneously in Canada, 2007.

BARROS, A.J.D.; HIRAKATA, V.N. Alternatives for logistic regression in crosssectional studies: an empirical comparison of models that directly estimate the prevalence ratio. BMC Med Res Methodol, v. 3, n. 21, p. 1-13, 2003.

BRASIL. Decreto no 1.948, de 3 de julho de 1996. Regulamenta a Lei 8.842, de 4 de janeiro de 1994, a qual dispõe sobre a Política Nacional do Idoso, e dá outras providências. Diário Oficial da União. 4 jul. 1996; Seção1:12277.
CHATTERJEE, S.; PRICE, B. Regression analysis by example. Fourth edition. New Jersey: John Wiley and Sons, Inc., 2006.

COLÓSIMO, E.A.; GIOLO, S.R. Análise de sobrevivência aplicada. 1ํe ed Ed: Edgard Blucher, 2006.

COOK, R. D.; WEISBERG, S. Diagnostics for heteroskedasticity in regression. Biometrika, v. 70, n. 1, p. 1-10, 1983.

COUTINHO, L.M.S.; SCAZUFCA, M.; MENEZES, P.R. Métodos para estimar razão de prevalência em estudos de corte transversal. Rev Saúde Pública, v. 42, n. 6, p. 992-998, 2008.

DAL RIO, M.C. Perspectiva social do envelhecimento. São Paulo: Fundação Padre Anchieta, 2009.

DIAS, L.D.; BRITO, G.E.G.; FORTE, F.D.S.; ARAÚJO, K.M.B.; LUCENA, E.M.F. Perfil sociodemográfico e de saúde de idosos do município de João Pessoa - PB. Rev Bras Promoç Saúde, v. 25, n. 1, p. 86-96, 2012.

DRAPER, N.R.; SMITH, H. Applied regression analysis. New York: John Wiley \& Sons, inc., 1998.

ESPINOSA, M.M.; RODRIGUES, D.C.; MARCON, S.R. Planejamento amostral probabilístico em estudos comparativos com grupos de idosos. Conection Line, v. 13, p.74-84, 2015.

FLECK, M.P.A.; CHACHAMOVICH, E.; TRENTINI, C. Development and validation of the Portuguese version of the WHOQOL-OLD module. Rev Saúde Pública, v. 40, n. 5, p. 785-91, 2006.

FLECK，M.P.A.; LOUZADA，S.; XAVIER，M.; CHACHAMOVICH, E.; VIEIRA, G.; SANTOS, L. Aplicação da versão em português do instrumento abreviado de avaliação da qualidade de vida "WHOQOL-BREF". Rev Saúde Pública, v. 34, n. 2 , p. 178-83, 2000.

FLECK MPA. WHOQOL-ABREVIADO. Versão em português dos instrumentos de avaliação de qualidade de vida (WHOQOL). Grupo de Estudos em Qualidade de Vida. Universidade Federal do Rio Grande do Sul, 1998.

FORTIN, M.; LAPOINTE, L.; HUDON, C.; VANASSE, A.; NTETU, A.L.; MALTAIS, D. Multimorbidity and quality of life in primary care: a systematic review. Health Qual Life Outcomes, v. 2, n.51, p. 1-12, 2004.

GUTIERREZ, B.A.O.; AURICCHIO, A.M.; MEDINA, N.V.J. Mensuração da qualidade de vida de idosos em centros de convivência. J Health Sci Inst, v. 29, p. 186-190, 2011.

JORGE, M.M. Idosos - Experiências de Inclusão. In: 19ª Conferência Mundial de Serviço Social; 2008; Salvador, BR. Bahia: Centro de Conferências Salvador; 2008. p.1-4. 
KUTNER, M.H.; NACHTSHEIM, C.J.; NETER, J. Applied Linear Statistical Models. Fifth edition. New York: Mc Graw Hill, Companies, Inc., 2005.

LE, C.T. Introductory Biostatistics. New Jersey: Published by John Wiley \& Sons, Inc., Hoboken. Published simultaneously in Canada, 2003.

LIN, D.Y.; WEI, L.J. The robust Inference for the Cox Proportional Hazards Model. J Am Stat Assoc., v. 84, n. 408, p. 1074-8, 1989.

Minitab 17 Statistical Software 2010. [Computer software]. State College, PA: Minitab, Inc. (www.minitab.com).

OLIVEIRA, N.S.; SOUZA, T.S.; ALENCAR, F.S.; OLIVEIRA, G.L.; FERREIRA, N.B.; ALENCAR, J.S. Percepção dos Idosos Sobre o Processo de Envelhecimento. Revista de Psicologia, v. 22, p. 40-83, 2014.

PAGOTTO, V.; KYOSEN, A.Y.; SILVEIRA, N.E.A. Fatores associados à autoavaliação de saúde ruim em idosos usuários do Sistema Único de Saúde. Cad Saúde Pública, v. 27, p. 1593-1602, 2011.

PAIVA, M.H.P.; PEGORARI, M.S.; NASCIMENTO, J.S.; SANTOS, A.S. Fatores associados à qualidade de vida de idosos comunitários da macrorregião do Triângulo do Sul, Minas Gerais, Brasil. Ciênc Saúde Coletiva, v. 21, n. 11, p. 3347-3356, 2016.

PEREIRA, R.J.; COTTA, R.M.M.; FRANCESCHINI, S.C.C.; RIBEIRO, R.C.L.; SAMPAIO, R.F.; PRIORE, S.E.; et al. Influência de fatores sociossanitários na qualidade de vida dos idosos de um município do Sudeste do Brasil. Ciênc Saúde Coletiva, v. 16, p. 2907-2917, 2011.

PEREIRA, R.J.; COTTA, R.M.M.; FRANCESCHINI, S.C.C.; RIBEIRO, R.C.L.; SAMPAIO, R.F.; PRIORE, S.E. et al. Contribuição dos domínios físico, social, psicológico e ambiental para a qualidade de vida global de idosos. Rev Psiquiatria, v. 28, p. 27-38, 2006.

RODRIGUES, C.D. de. Influência do Centro de Convivência na qualidade de vida de idosos: estudo comparativo. 2013. Dissertação de mestrado. Cuiabá: Instituto de Saúde Coletiva da Universidade Federal de Mato Grosso, 2013. $132 \mathrm{p}$.

SANTOS, K.A.; KOSZUOSKI, R.; COSTA, J.S.D.; PATTUSSI, M.P. Fatores associados com a incapacidade funcional em idosos do Município de Guatambu, Santa Catarina, Brasil. Cad Saúde Pública, v. 23, p. 2781-2788, 2007.

SCHNEIDER, R.H.; IRIGARY, T.Q. O envelhecimento na atualidade: aspectos cronológicos, biológicos, psicológicos e sociais. Estudos de Psicologia, v. 25, n. 4, p. 585-593, 2008.
SPILKER, B. Introduction. In: Spilker B (ed). Quality of Life Assessments in Clinical Trials. New York: Raven Press, 1990. p. 3-10.

IBM Corp. Released 2011. SPSS Statistics for Windows, version 20.0. Armonk, NY: IBM Corp.

TAVARES, D.M.S.; GOMES, N.C.; DIAS, F.A; Santos, N.M.F. Fatores associados à qualidade de vida de idosos com osteoporose residentes na zona rural. Esc Anna Nery, v. 16, n. 2, p. 371-378, 2012.

TAVARES, D.M.S.; MATIAS, T.G.C.; FERREIRA, P.C.S.; PEGORARI, M.S.; NASCIMENTO, J.S.; PAIVA, M.M. Qualidade de vida e autoestima de idosos na comunidade. Ciênc Saúde Coletiva, v. 21, n. 11, p. 3557-64, 2016.

TRENTINI, C.M. Qualidade de vida em idosos. 2004. Tese de doutorado. Porto Alegre: Faculdade de Medicina da UFRGS; 2004. 224p.

VAGETTI, G.C.; OLIVEIRA, V.; BARBOSA, F.V.C.; MOREIRA, N.B.; CAMPOS, W. Predição da qualidade de vida global em idosas ativas por meio dos domínios do WHOQOL-BREF e do WHOQOL-OLD. Motricidade, v.8, n. 2, p. 709-718, 2012.

VAGETTI, G.C.; BARBOSA, F.V.C.; MOREIRA, N.B.; OLIVEIRA, V.; MAZZARDO, O.; CAMPOS, W. Condições de saúde e variáveis sociodemográficas associadas à qualidade de vida em idosas de um programa de atividade física de Curitiba, Paraná, Sul do Brasil. Cad. Saúde Pública, v.29, n. 5, p. 955-969, 2013.

WHO -World Health Organization. Promoción de la salud. Glosario. Geneva, 1998.

WHOQOL GROUP. The World Health Organization quality of life assessment. (WHOQOL): Position paper from the World Health Organization. Soc. Sci. Med., v. 41, p. 1403-1409, 1995.

WHOQOL GROUP. The World Health Organization quality of life assessment (WHOQOL): Development and general psychometric properties. Soc Sci Med, v. 46, p. 1569-1585, 1998. 


\section{Contribuição dos autores}

Mariano Martinez Espinosa

Concepção e desenho do projeto da pesquisa, análise e interpretação dos dados, análise estatística, redação e revisão crítica relevante do manuscrito e do conteúdo intelectual.

\section{Nathany Luzia de Oliveira}

Processamento, análise e interpretação dos dados, redação do manuscrito e análise e revisão crítica do manuscrito.

\section{Dayane de Carvalho Rodrigues}

Concepção e desenho da pesquisa, coleta de dados, redação do manuscrito e revisão crítica relevante do conteúdo intelectual.

\section{Bryan Mariano Martínez Alves}

Processamento, análise e interpretacão dos dados, análise estatística, redação do manuscrito e revisão crítica do manuscrito quanto ao conteúdo intelectual importante.

\section{Samira Reschetti Marcon}

Revisão crítica relevante do manuscrito, participação ativa na

discussão dos resultados e aprovação final da versão a ser publicada e concordância com todos os aspectos do manuscrito em termos de veracidade ou integridade das informações. 